\title{
Diagnostics of supercapacitors
}

\author{
Valeriy Martynyuk ${ }^{1 *}$, Oleksander Eromenko ${ }^{1}$, Juliy Boiko ${ }^{1}$, Tomasz Kałaczyński ${ }^{2}$ \\ ${ }^{1}$ Programming and Computer, Telecommunication Systems Faculty in Khmelnytsky National \\ University, Department of Telecommunication and Computer Integrated Technology, 29016, 11 \\ Institutska street, Khmelnytsky, Ukraine \\ ${ }^{2}$ Faculty of Mechanical Engineering UTP in Bydgoszcz, Department of Vehicle Engineering, Al. \\ Prof. S.Kaliskiego 7, 85-796 Bydgoszcz, Poland
}

\begin{abstract}
The paper represents the mathematical model for diagnostics of supercapacitors. The research objectives are the problem of determining a supercapacitor technical condition during its operation. The general reliability of diagnostics is described as the methodological and instrumental reliabilities of diagnostics. The instrumental diagnostic reliability of supercapacitor includes the probabilities of errors of the first and second kind, $\alpha$ and $\beta$ respectively. The methodological approach to increasing the reliability of supercapacitor diagnostic has been proposed, in terms of multiparameter supercapacitor diagnostic by applying nonlinear, frequency dependent mathematical models of supercapacitors that take into account nonlinearity, frequency dispersion of parameters and the effect of transient processes in supercapacitors. The more frequencies, operating voltages and currents are applied in the supercapacitor diagnostics, the more methodological reliability of diagnostics will increase in relation to the methodological reliability of supercapacitor diagnostics when only one frequency, voltage and current are applied.
\end{abstract}

\section{Introduction}

Supercapacitors are polar electrochemical devices that are capable of storing and releasing electrical energy by internal redistribution of electrolyte ions. According to their electrical parameters, supercapacitors occupy an intermediate position between electrolytic capacitors of high capacitance and batteries, but according to the principle of operation differ from both of them $[1,2]$.

The quality and reliability of supercapacitors are largely determined by diagnostics support at all stages of their life cycle. At the production stages, where it is crucial to ensure zero-defect manufacturing technology and labour for producing supercapacitors, the volume of control and diagnostic operations reaches $50 \%$ of the total labour intensity of their production. A characteristic problem of diagnosing supercapacitor manufacturing defects under the conditions of their mass production is that the later the defects are detected, the more costs are necessary to localize the place of their emergence.

Hence, diagnostic support will be effective only when the high reliability values are ensured at minimum cost. At the operation stage, the purpose of diagnosing a superapacitor is to evaluate its technical condition, to determine the reasons of its operation failures, to predict changes in a supercapacitor technical condition and determine the reasons for such changes or time intervals after which processes causing undesirable changes in a supercapacitor technical condition might begin.

At present, the scope of supercapacitor application is very wide, namely electric vehicles, hybrid transport, devices for starting diesel and gasoline internal combustion engines,

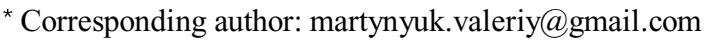


powerful electronics and telecommunications. Thus, the issues of developing methods and means of supercapacitor diagnostics have become urgent.

In most cases, supercapacitors are applied in parallel with chemical elements, batteries and accumulators for smoothing the peak values of load current, which greatly increases operation lifetime and improves the characteristics of electrochemical power sources.

Each year, supercapacitor mass production increases and we observe gradual improvement of the electrical parameters of supercapacitors that are produced by world leading companies such as Maxwell, AVX, Cooper, Elna (USA), Fuji Heavy Industries, NEC, Panasonic, Power Systems (Japan), Ness, LS Cable (South Korea), EPCOS (Germany), BatScap (France), Cap-XX (Australia), Tavrima (Canada), ESMA, EL Technology (Russia) and Yunasko (Ukraine).

The best samples of modern supercapacitors are characterized by capacities in thousands of Farads, high operating currents (hundreds and thousands of amperes), and low values of active resistance (several milliohms and hundreds of microhms).

\section{Supercapacitor technical condition}

Supercapacitor diagnostics is an information procedure the purpose of which is to reflect its technical condition in the form of a conclusion about the nature and significance of this condition. Such a conclusion regarding the diagnostic results is called a diagnosis [3].

Technical condition of a supercapacitor is a condition that is characterized at a certain time, under certain environmental conditions by the values of supercapacitor parameters set by its data sheet. According to the existing practice, it has been confirmed to define such mutually exclusive incompatible pairs of technical conditions for technical products: serviceable - unserviceable, operable - faulty.

For the serviceable - unserviceable conditions the basis of their comparison is compliance of all supercapacitor parameters with the requirements of normative data sheet. And if at least one of these parameters, regardless of its impact on the supercapacitor ability to perform its functions, will not fulfill these requirements, the technical condition is considered unserviceable. And when all the parameters meet all the requirements of proper documentation, the technical condition is serviceable.

A supercapacitor is in an operable technical condition if its relevant diagnostic parameters are within the limits where working functions can be performed. When the diagnostic parameters go beyond the specified limits, then the supercapacitor can no longer fully perform its functions, and it has to be considered faulty.

Evaluation of a supercapacitor technical condition is a complex task that allows solving the following problems:

- at the production stage - preventing disruption of technological processes;

- at the operation stage - detecting emerging defects at an early stage, thus preventing processes that would lead to undesirable change in a supercapacitor technical condition and cause emergency situations.

Principally, the problem of determining a supercapacitor technical condition can be solved in two ways: functional and test diagnostics. While performing functional diagnostics it is necessary to observe the behavior of an operating supercapacitor and compare it with the standard. For test diagnostics it is necessary to compile a description of a supercapacitor for various types of its malfunctions and to determine what influences they can be detected by.

Low reliability of supercapacitor diagnostics in the existing diagnostic systems is conditioned by a complex change in diagnostic parameters in case of supercapacitor malfunction. This circumstance does not permit to fully solve the problem of analyzing a supercapacitor technical condition neither in static nor in dynamic operating modes at the supercapacitor production and operation stages. 
Reliability of diagnostics is the degree of objective conformity of the result of diagnostics to the actual technical condition of the supercapacitor, which is estimated by the probability of making the correct decision about the condition of the supercapacitor [4]. The components of the general reliability of diagnostics are methodological and instrumental reliabilities of diagnostics [5]:

$$
D_{G}=D_{M} \cdot D_{I},
$$

where $D_{M}$ is methodological reliability of diagnostics; $D_{I}$ is instrumental reliability of diagnostics.

Methodological reliability of diagnostics is calculated by the following formula [5]:

$$
D_{M}=\frac{N_{1}}{N}
$$

where $N_{1}$ is the number of exponents which characterize the technical condition of the supercapacitor and are included into the mathematical model of diagnostics;

$\mathrm{N}$ is total number of exponents which characterize the diagnostics of the supercapacitor.

Instrumental reliability of diagnostics is defined by the probabilities of errors of the first and second kind respectively $\alpha$ i $\beta[5]$ :

$$
D_{I}=1-\alpha-\beta
$$

where $\alpha$ is probability of errors of the first kind; $\beta$ is probability of errors of the second kind.

\section{Mathematical model for diagnostics of supercapacitors}

Let us consider a simplified mathematical model for diagnostics of supercapacitors in the stationary mode, which is based on a one-time measurement of capacitance $\mathrm{C}$, active resistance $\mathrm{R}$ and self-discharge current Isd of supercapacitors.

Depending on the diagnostic method, capacitance $\mathrm{C}$ and active resistance $\mathrm{R}$ of supercapacitors are measured either at the alternating current at one probe effect frequency $\omega 0$ with one nominal constant voltage at the supercapacitor outputs Unom or at the direct charge (discharge) current of supercapacitors $\mathrm{I}_{0}$. Self-discharge current Isd of supercapacitors is also measured at one nominal constant voltage at the supercapacitor outputs Unom. As a result of such measurements, we get three measured values: $r$, $c$ and isd.

Next, two hypotheses are put forward.

1. The basic hypothesis $\mathrm{H}_{0}$ (the supercapacitor is working):

$$
H_{0}: r \in\left[R_{\min }, R_{\max }\right] \cap c \in\left[C_{\min }, C_{\max }\right] \cap i_{s d} \in\left[I s d_{\min }, I s d_{\max }\right],
$$

where $\mathrm{R}_{\min }, \mathrm{R}_{\max } ; \mathrm{C}_{\min }, \mathrm{C}_{\max }$ and $\mathrm{Isd}_{\min }$, Isd $\mathrm{I}_{\max }$ are limits of permissible interval for $\mathrm{r}, \mathrm{c}$ and $i_{\text {sd }}$ respectively.

2. Alternative hypothesis $\mathrm{H}_{1}$ (the supercapacitor is defective):

$$
H_{1}: r \notin\left[R_{\min }, R_{\max }\right] \cap c \notin\left[C_{\min }, C_{\max }\right] \cap i_{s d} \notin\left[I s d_{\min }, I s d_{\max }\right] .
$$

The diagnostic reliability of the supercapacitor is defined as the product of the methodological and the instrumental diagnostic reliabilities of supercapacitor (1). The methodological diagnostic reliability of supercapacitor is defined by the ratio between the number of exponents characterizing the technical condition of the object of diagnostic and included to the mathematical model of diagnostics and the total number of exponents characterizing the technical condition of the supercapacitor.

From this we can conclude that the simplified mathematical model for diagnostics of supercapacitors, which is based on the one-time measurement of capacitance $\mathrm{C}$, active resistance $\mathrm{R}$ and self-discharge current Isd of supercapacitors, is characterized by low methodological reliability of diagnostics.

It can be explained by the fact that for supercapacitors capacitance $\mathrm{C} \neq$ const, active resistance $\mathrm{R} \neq$ const and self-discharge current Isd $\neq$ const. 
In case of a simplified diagnostic model of supercapacitor, the number of exponents characterizing the technical condition of supercapacitors $\mathrm{N}_{1}=3$ (capacitance, active resistance and self-discharge current). As for the total number of exponents characterizing the technical condition of supercapacitors, frequency dispersion and non-linearity of supercapacitor parameters $(\mathrm{R}(\omega, \mathrm{u}), \mathrm{C}(\omega, \mathrm{u})$, Isd $(\mathrm{t}, \mathrm{u}))$ are equivalent to the increase in the total number of exponents characterizing the technical condition of supercapacitors. Hence, we can conclude that $\mathrm{N} \neq \mathrm{N} 1$ i $\mathrm{N} \gg>\mathrm{N} 1$, and the methodological diagnostic reliability of supercapacitor for a simplified model of supercapacitor diagnostics is much smaller than one.

$$
D_{M}=\frac{3}{N}<<1
$$

The instrumental diagnostic reliability of supercapacitor is defined by the expression (3), which includes the probabilities of errors of the first and second kind, $\alpha$ and $\beta$ respectively. The probabilities of errors of the first and second kind are defined separately for the capacitance $\mathrm{C}$, active resistance $\mathrm{R}$ and self-discharge current Isd of supercapacitors according to the expressions:

$$
\begin{aligned}
& \alpha_{C}=\int_{C_{\min }}^{C_{\max }} f(C)\left(\int_{-\infty}^{C_{\min }-C} f\left(\delta_{C}\right) d \delta_{C}\right) d C+\int_{C_{\min }}^{C_{\max }} f(C)\left(\int_{C_{\max }-C}^{\infty} f\left(\delta_{C}\right) d \delta_{C}\right) d C ; \\
& \beta_{C}=\int_{-\infty}^{C_{\min }} f(C)\left(\int_{C_{\min }-C}^{C_{\max }-C} f\left(\delta_{C}\right) d \delta_{C}\right) d C+\int_{C_{\max }}^{\infty} f(C)\left(\int_{C_{\min }-C}^{C_{\max }-C} f\left(\delta_{C}\right) d \delta_{C}\right) d C,
\end{aligned}
$$

where $C_{\max }, C_{\min }$ are permissible maximal and minimal capacitance values for supercapacitors; $f(C)$ is density of probability of supercapacitor capacitance; $f\left(\delta_{C}\right)$ is density of probability of supercapacitor capacitance measurement error.

$$
\begin{aligned}
& \alpha_{R}=\int_{R_{\min }}^{R_{\max }} f(R)\left(\int_{-\infty}^{R_{\min }-R} f\left(\delta_{R}\right) d \delta_{R}\right) d R+\int_{R_{\min }}^{R_{\max }} f(C)\left(\int_{R_{\max }-R}^{\infty} f\left(\delta_{R}\right) d \delta_{R}\right) d R ; \\
& \beta_{R}=\int_{-\infty}^{R_{\max }} f(R)\left(\int_{R_{\min }-R}^{R_{\max }-R} f\left(\delta_{R}\right) d \delta_{R}\right) d R+\int_{R_{\max }}^{\infty} f(R)\left(\int_{R_{\min }-R}^{R_{\max }-R} f\left(\delta_{R}\right) d \delta_{R}\right) d R,
\end{aligned}
$$

where $R_{\max }, R_{\min }$ is permissible maximal and minimal active resistance values for supercapacitors; $f(R)$ is density of probability of supercapacitor active resistance; $f\left(\delta_{R}\right)$ is density of probability of supercapacitor active resistance measurement error.

$$
\begin{aligned}
& \alpha_{I s d}=\int_{I s d_{\min }}^{I s d_{\max }} f(I c p)\left(\int_{-\infty}^{I s d_{\min }-I s d} f\left(\delta_{I s d}\right) d \delta_{I s d}\right) d I s d+ \\
& +\int_{I s d_{\min }}^{I s d_{\max }} f(I c p)\left(\int_{I s d_{\max }-I s d}^{\infty} f\left(\delta_{I s d}\right) d \delta_{I s d}\right) d I s d \\
& \beta_{I s d}=\int_{-\infty}^{I s d_{\min }} f(R)\left(\int_{I s d_{\max }-I s d}^{I s d_{\max }-I s d} f\left(\delta_{I s d}\right) d \delta_{I s d}\right) d I s d+ \\
& +\int_{I s d_{\max }}^{\infty} f(I s d)\left(\int_{I s d_{\min }-I s d}^{I s d_{\max }-I s d} f\left(\delta_{I s d}\right) d \delta_{I s d}\right) d I s d
\end{aligned}
$$


where $I s d_{\max }, I s d_{\min }$ are permissible maximal and minimal self-discharge current values for supercapacitors; $f(I s d)$ is density of probability of supercapacitor self-discharge current; $f\left(\delta_{\text {Isd }}\right)$ is density of probability of supercapacitor self-discharge current measurement error.

The general probabilities of errors of the first and second kind, $\alpha$ and $\beta$ respectively, are defined by the expressions:

$$
\begin{aligned}
& \alpha=1-\left(1-\alpha_{C}\right)\left(1-\alpha_{R}\right)\left(1-\alpha_{\text {Isd }}\right) ; \\
& \beta=1-\left(1-\beta_{C}\right)\left(1-\beta_{R}\right)\left(1-\beta_{\text {Isd }}\right) .
\end{aligned}
$$

Then, the general diagnostic reliability of supercapacitor for a simplified model is defined by the expression:

$$
D_{G}=D_{M} \cdot D_{I}=\frac{3}{N} \cdot(1-\alpha-\beta)<<1 .
$$

Assessment of supercapacitor diagnostics for a simplified model can also be performed using the formula of average diagnostic risk:

$$
\Psi \approx W_{1 R} \alpha_{R}+W_{2 R} \beta_{R}+W_{1 C} \alpha_{C}+W_{2 C} \beta_{C}+W_{1 I s d} \alpha_{I s d}+W_{2 I s d} \beta_{I s d},
$$

where $\mathrm{W}_{1 \mathrm{R}}, \mathrm{W}_{1 \mathrm{C}}$ and $\mathrm{W}_{1 \mathrm{Isd}}$ are losses due to errors of the first kind for each parameter $\mathrm{r}$, $c$ and $i_{\text {sd }}$ respectively;

$\mathrm{W}_{2 \mathrm{R}}, \mathrm{W}_{2 \mathrm{C}}$ and $\mathrm{W}_{2 \mathrm{Isd}}$ are due to errors of the second kind for each parameter $\mathrm{r}$, $\mathrm{c}$ and $\mathrm{i}_{\mathrm{sd}}$ respectively;

$\alpha_{R}, \alpha_{C}$ and $\alpha_{\text {Isd }}$ are probabilities of errors of the first kind for each parameter $r, c$ and $i_{s d}$ respectively;

$\beta_{\mathrm{R}}, \beta_{\mathrm{C}}$ and $\beta_{\text {Isd }}$ are probabilities of errors of the second kind for each parameter $r, c$ and $i_{s d}$ respectively.

Increase in the diagnostic reliability of supercapacitor is possible when using supercapacitor characteristics that contain information on the nominal values of technical condition of supercapacitors in the range of changes in nominal voltages in the operation frequency range or in the operation time range. Such characteristics include nonlinear complex resistance $\mathrm{Z}(\mathrm{j} \omega, \mathrm{u})$ (or nonlinear resistance $Z(s, u)$ and nonlinear transient resistance $z(t, u)$ of supercapacitors, related to each other by inverse and direct Laplace transforms:

$$
\begin{aligned}
& Z(s, u)=L^{-1}[z(t, u)] ; \\
& z(t, u)=L[Z(s, u)],
\end{aligned}
$$

where $s=j \omega$ is complex frequency.

The supercapacitor's input is supplied with either the probe voltage $u_{p}(t)$ or probe current $i_{p}(t)$, and either the current $i_{m}(t)$ through the supercapacitor or the voltage $u_{m}(t)$ at the supercapacitor is measured. Operators $Z$ or $Y$ determine the complex of mathematical operations over $u_{p}(t)$ or $i_{p}(t)$ which allow obtaining $i_{m}(t)$ or $u_{m}(t)$ respectively:

$$
\begin{aligned}
& i_{m}(t)=Z\left[u_{p}(t)\right] ; \\
& u_{m}(t)=Y\left[i_{p}(t)\right] .
\end{aligned}
$$

Let's consider that the probe voltage $u_{p}(t)$ and the probe current $i_{p}(t)$ are observed without an error, and the measured voltage $u_{m}(t)$ and the measured current $i_{m}(t)$ are observed with errors:

$$
i_{m}^{*}(t)=i_{m}(t)+\Delta i_{m}(t)
$$




$$
u_{m}^{*}(t)=u_{m}(t)+\Delta u_{m}(t) .
$$

where $\Delta i_{m}(t)$ and $\Delta u_{m}(t)$ are stationary random processes with known characteristics.

Hence, we can conclude that in the process of supercapacitor diagnostics it is necessary to obtain a rating of the defined operators $Z_{m}$ and $Y_{m}$ approaching the operators $Z$ and $Y$ which describe the properties of supercapacitors. Taking into account the fact that operators $Z$ or $Y$ are in most cases unknown, to describe supercapacitors their mathematical models characterized by operators $Z_{\text {mod }}$ or $Y_{\text {mod }}$ are used [6,7].

The criterion for estimating the proximity of operators $Z_{m}, Y_{m}$, and $Z_{\text {mod }}, Y_{\text {mod }}$ is approximation of the measured voltage $u_{m}(t)$ and the measured current $i_{m}(t)$ to the voltage $u_{\text {mod }}(t)$ and current $i_{\text {mod }}(t)$ of the model at the similar probe effects of $i_{p}(t)$ and $u_{p}(t)$ respectively.

Let's introduce positive functions of losses $\eta_{u}\left[u_{m}(t), u_{\text {mod }}(t)\right]$ or $\eta_{i}\left[i_{m}(t), i_{\text {mod }}(t)\right]$ which depend on the measured voltage and current of an supercapacitor and its model, and also determine the amount of losses connected with different combinations of $u_{m}(t), u_{\text {mod }}(t)$ and $i_{m}(t), i_{\bmod }(t)$. Average loss or average risk is defined by the mathematical expectations of loss functions $\eta_{u}\left[u_{m}(t), u_{\text {mod }}(t)\right]$ or $\eta_{i}\left[i_{m}(t), i_{\bmod }(t)\right]$ :

$$
\begin{aligned}
& \bar{\Psi}_{u}=M\left\{\eta_{u}\left[u_{m}(t), u_{\mathrm{mod}}(t)\right]\right\} ; \\
& \bar{\Psi}_{i}=M\left\{\eta_{i}\left[i_{m}(t), i_{\mathrm{mod}}(t)\right]\right\} .
\end{aligned}
$$

On the other hand, average risks are defined by expressions:

$$
\begin{aligned}
& \bar{\Psi}_{u}=\iint \eta_{u}\left(u_{m}, u_{\mathrm{mod}}\right) f_{u}\left(u_{m}, u_{\mathrm{mod}}\right) d u_{m} d u_{\mathrm{mod}} \\
& \bar{\Psi}_{i}=\iint \eta_{i}\left(i_{m}, i_{\mathrm{mod}}\right) f_{i}\left(i_{m}, i_{\mathrm{mod}}\right) d i_{m} d i_{\mathrm{mod}},
\end{aligned}
$$

where $f_{u}\left(u_{m}, u_{\text {mod }}\right)$ and $f_{i}\left(i_{m}, i_{\text {mod }}\right)$ are voltage and current probability functionals.

Expressions (25) and (26) can be simplified, if the nonlinear transient resistance $z(t, u)$ and nonlinear transient conductance $y(t, i)$ of supercapacitors are monotone functions, and probe signals $u(t)$ and $i(t)$ are stationary:

$$
\begin{array}{r}
\bar{\Psi}_{u}=\int_{u \in U} \eta_{u}\left[z_{m}(t, u), z_{\mathrm{mod}}(t, u)\right] f_{u}(u) d u \\
\bar{\Psi}_{i}=\int_{i \in I} \eta_{i}\left[y_{m}(t, i), y_{\bmod }(t, i)\right] f_{i}(i) d i,
\end{array}
$$

where $U$ is definition domain of $u$;

$I$ is definition domain of $i$.

Deviation of the defined nonlinear transient resistance $z_{m}(t, u)$ from the nonlinear transient resistance of the model $z_{\text {mod }}(u)$, as well as deviation of the defined nonlinear transient conductance $y_{m}(t, i)$ from the nonlinear transient conductance of the model $y_{\text {mod }}(t, i)$ may be estimated by the criterion of regular approximation (maximum deviation of characteristics):

$$
\begin{aligned}
& m_{u}=\max _{u \in U}\left|z_{m}(t, u)-z_{\text {mod }}(t, u)\right| \\
& m_{i}=\max _{i \in I}\left|y_{m}(t, i)-y_{\text {mod }}(t, i)\right|
\end{aligned}
$$


Let's choose such mathematical models of supercapacitors $z_{\text {mod }}(t, u)$ and $y_{\bmod }(t, i)$ which will satisfy the criteria of regular approximation in the ranges of change in the probe voltage $u_{p}(t)$ and current $i_{p}(t)$ of supercapacitors.

Let's define the average risk of supercapacitor control for the following loss functions

$$
\begin{gathered}
\eta_{u}=\left|z_{m}(t, u)-z_{\text {mod }}(t, u)\right|^{k} \text { and } \eta_{i}=\left|y_{m}(t, i)-y_{\text {mod }}(t, i)\right|^{k}: \\
\bar{\Psi}_{u}=\int_{u \in U}\left|z_{m}(t, u)-z_{\bmod }(t, u)\right|^{k} f_{u}(u) d u \leq \int_{u \in U} m_{u}^{k} f_{u}(u) d u=m_{u}^{k} ; \\
\bar{\Psi}_{i}=\int_{i \in I}\left|y_{m}(t, i)-y_{\bmod }(t, i)\right|^{k} f_{i}(i) d i \leq \int_{i \in I} m_{i}^{k} f_{i}(i) d i=m_{i}^{k} .
\end{gathered}
$$

The advantage of the obtained expressions of the average supercapacitor diagnostic risks (31) and (32) in comparison with the expressions (25) and (26) is the possibility of calculating the maximum values of the average supercapacitor diagnostic risks that do not depend on the probability densities $f_{u}(u)$ and $f_{i}(i)$ of supercapacitor voltage and current probe effects.

\section{Defining reliability of supercapacitor diagnostics}

Let's consider the errors in supercapacitor diagnostics applying the developed supercapacitor diagnostics means. The law of changing supercapacitor capacitance, active resistance and self-discharge current depends on many factors, such as technological inaccuracy in manufacturing mechanisms involved in the production of electrodes, instability in time of the force applied to press the shafts, with the help of which the layer of activated carbon is applied to the aluminum foil of the electrodes.

It happens due to the vibration of equipment during the operation process, as well as changeable linear velocity of aluminum foil when it passes through the application area. It is difficult to distinguish a dominant one among all these external factors.

Relying on the analysis of the external factors affecting supercapacitor production, it has been found out that the laws of distribution of supercapacitor diagnostic parameters are normal and may be expressed as follows:

$$
\begin{array}{r}
f(C)=\frac{1}{\sigma_{C} \sqrt{2 \pi}} e^{\left(\frac{C-M_{C}}{2 \sigma_{C}^{2}}\right),} \\
f(R)=\frac{1}{\sigma_{R} \sqrt{2 \pi}} e^{\left(\frac{R-M_{R}}{2 \sigma_{R}^{2}}\right)}, \\
f(I s d)=\frac{1}{\sigma_{I s d} \sqrt{2 \pi}} e^{\left(\frac{I s d-M_{I s d}}{2 \sigma_{I s d}}\right)},
\end{array}
$$

where $\sigma_{C}, \sigma_{R}$ and $\sigma_{I c p}$ are mean squared deviations of capacitance value $C$, active resistance value $R$ and self-discharge current value $I s d$ of supercapacitor; $M_{C}, M_{R}$ and $M_{I s d}$ are nominal values of capacitance $C$, active resistance $R$ and self-discharge value Icp of supercapacitor.

Using the Mathcad symbolic mathematics package and expressions (33) - (35), dependencies of the first-order errors and the second-order errors on such parameters as $A_{C}=\sigma_{\tau C} / \sigma_{C}, A_{R}=\sigma_{\tau R} / \sigma_{R}$ and $A_{\text {Isd }}=\sigma_{\tau I s d} / \sigma_{\text {Isd }}$. have been calculated separately for capacitance $\mathrm{C}$, active resistance $\mathrm{R}$ and self-discharge current $I s d$ of supercapacitor. 
These parameters determine the ratio between the mean squared deviation of the total measurement error of capacity $C$, active resistance $R$ and self-discharge current $I s d$ of supercapacitor and mean squared deviation of these parameters.

In the calculations, it was assumed that the control increments of the tolerance zones for the upper and lower limits are zero and tolerance zones for capacitance $C$, active resistance $R$ and self-discharge current $I s d$ of supercapacitor were considered equal to $5 \%$ of their nominal values. The nominal values of capacitance $C$, active resistance $R$ and selfdischarge current $I s d$ of supercapacitor were considered equal to $C=100 \mathrm{~F}, R=7 \mathrm{mOhm}$ and $I s d=0,1 \mathrm{~mA}$ correspondingly.

The general probabilities of supercapacitor diagnostic errors of the first and the second order $-\alpha$ and $\beta$ correspondingly - are defined by the expressions (7) and (12). The graphs of dependencies between the diagnostic errors of the first and the second order $-\alpha$ and $\beta$ correspondingly - are shown in the fig. 1 ( $a$ and $b$, respectively).

a)
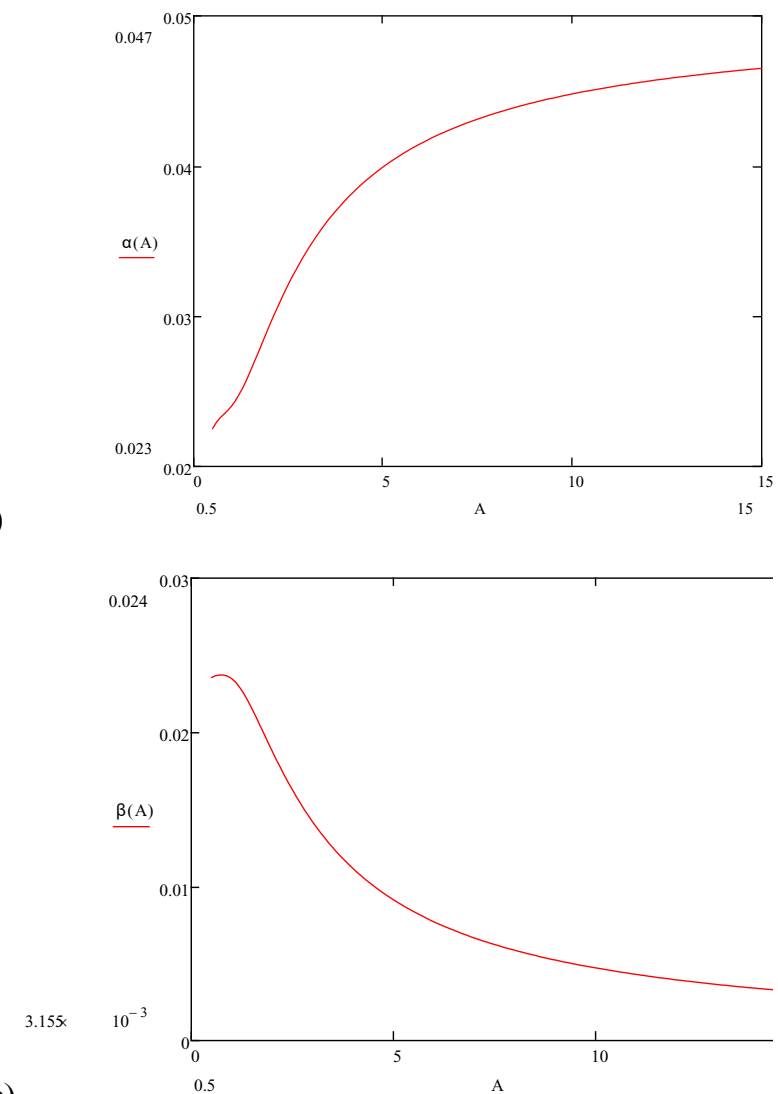

b)

a) graph of dependency between the first-order diagnostic error $\alpha$ and parameter $\mathrm{A}$;

b) graph of dependency between the second-order diagnostic error $\beta$ and parameter $A$

Fig. 1. Graphs of dependency between the first-order $\alpha$ the second-order $\beta$ diagnostic errors and parameter A.

The instrumental reliability of supercapacitor diagnostics is determined by the errors of the first and second orders $\alpha$ and $\beta$ according. The probability of making an erroneous decision in supercapacitor diagnostics will be equal to the sum of errors of the first and the second orders. The graphs of dependencies between the instrumental reliability of 
supercapacitor diagnostics and the probability of making an erroneous decision in the process of supercapacitor diagnostics and parameter A, are shown in fig. 2 ( $a$ and b, respectively).

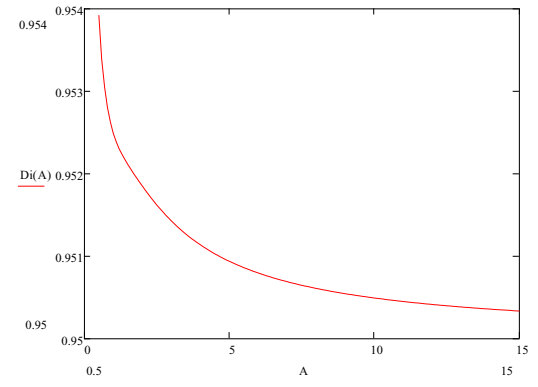

a)

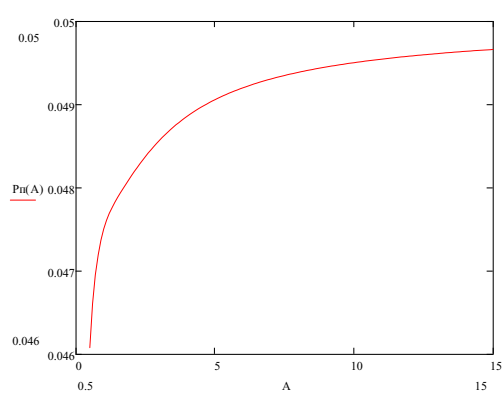

b)

a) graph of dependency between the instrumental reliability of diagnostics $D_{i}$ and parameter A; b) graph of dependency between the probability of making an erroneous decision $P_{\Pi \Pi}$ and parameter A

Fig. 2. Graphs of dependencies between the instrumental reliability of diagnostic, the probability of making an erroneous decision and parameter $\mathrm{A}$.

Analysis of the dependency between the instrumental reliability of diagnostics, which represents the probability of a correct decision for the above-set parameters, varies from 0,954 to 0,95 .

Methodological reliability of diagnostics is determined by the ratio between the number of parameters that characterize technical condition of the object of control being included into the mathematical model of diagnostics and the total number of parameters that characterize technical condition of the object of diagnostics.

If the number of parameters that characterize technical condition of the object of diagnostics being included into the mathematical model of diagnostics is equal to the total number of parameters that characterize technical condition of the object of diagnostics, then methodological reliability of diagnostics is equal to one, and the total reliability of diagnostics will be equal to the instrumental reliability of diagnostics:

$$
D_{G}=D_{M} \cdot D_{I}=0,95 \text {. }
$$

In the case of using only one frequency, voltage and current, at which supercapacitor diagnostics is carried out, the methodological reliability of diagnostics sharply decreases and is defined by the expression

$$
D_{M O}=\frac{3}{N} .
$$

The more frequencies, operating voltages and currents are applied in the supercapacitor diagnostics, the more methodological reliability of diagnostics will increase in relation to the methodological reliability of supercapacitor diagnostics when only one frequency, voltage and current are applied. Increase in the methodological reliability of supercapacitor diagnostics while applying various frequencies, operating voltages and control currents can be defined by the expression

$$
\mathrm{K}=\frac{D_{M F}}{D_{M O}}=\frac{L}{3},
$$

where $D_{M F}$ is methodological reliability of supercapacitor diagnostics while applying $L$ frequencies, operating voltages and currents.

\section{Conclusions}


Analysis of the expressions of the average supercapacitor diagnostic risks (31) and (32) shows that development of mathematical models of supercapacitors that most accurately describe their parameters and characteristics is extremely important for reducing the average supercapacitor diagnostic risks.

The methodological approach to increasing the reliability of supercapacitor diagnostic has been proposed, in terms of multi-parameter supercapacitor diagnostic by applying nonlinear, frequency dependent mathematical models of supercapacitors that take into account nonlinearity, frequency dispersion of parameters and the effect of transient processes in supercapacitors.

The procedure for increasing the reliability of supercapacitor diagnostic has been suggested on the basis of minimizing the average supercapacitor diagnostic risks that do not depend on the probability densities of probe effects of supercapacitor voltage and current.

\section{References}

1. Conway B. E. Electrochemical Supercapacitors: Scientific Principles and Technological Application / B. E. Conway. - New York : Plenum, 1999. - P. 435

2. Martynyuk V. V. Fractional Model of an Electrochemical Capacitor / V. V. Martynyuk, M. D. Ortigueira // Journal of Signal Processing. - V. 107, 2015, pp. 355-360

3. IEC Publication 50 (191) June 1988: Chapter 191 of the IEV: Reliability, maintainability and quality of service

4. Radford, George S. The Control of Quality in Manufacturing, New York: Ronald Press Co

5. D. C. Montgomery and G.C. Runger, Applied Statistics and Probability for Engineers. 2nd Edition, John Wiley \& Sons, New York, 1999

6. Martynyuk V., Vdovin O., Boyko J. and. Vlasenko N. "Super-high capacitor analyzer with compensation of common-mode error," in Proc. IMECO TC-4, 2001, pp. 340-343

7. Martynyuk V., Makaryshkin D., Boyko J. "Frequency Domain Analysis for Electrochemical Supercapacitors" in Proc. IMEKO TC-4, 2007, pp. 357-361 